\title{
Fever, hepatosplenomegaly and pancytopenia in a patient living in the Mediterranean region
}

\author{
Yahya Büyükaşık, N Şemnur İleri, İ Celalettin Haznedaroğlu, Halûk Demiroğlu, \\ Semra Dündar
}

A 24-year-old woman living in the Mediterranean region of Turkey presented with a three-month history of weight loss and irregular fever that was peaking at $40^{\circ} \mathrm{C}$ with shivering. No definite aetiology could be identified in a local hospital. A bacterial infection had been suspected, but antibiotic therapy, at first with sulbactam-ampicillin and later with azithromycin, had no influence on the fever. Physical examination revealed an emaciated patient with fever $\left(39^{\circ} \mathrm{C}\right)$, pallor, and hepatosplenomegaly (spleen $9 \mathrm{~cm}$ and liver $5 \mathrm{~cm}$ palpable below the costal margin). No peripheral lymphadenopathy was present. The laboratory examinations are summarised in the table. Notably, a prominent increase of macrophages containing intracellular micro-organisms (figures 1 and 2) was seen in the bone marrow smears. The same micro-organisms were also identified within the Kupffer cells in liver biopsy.
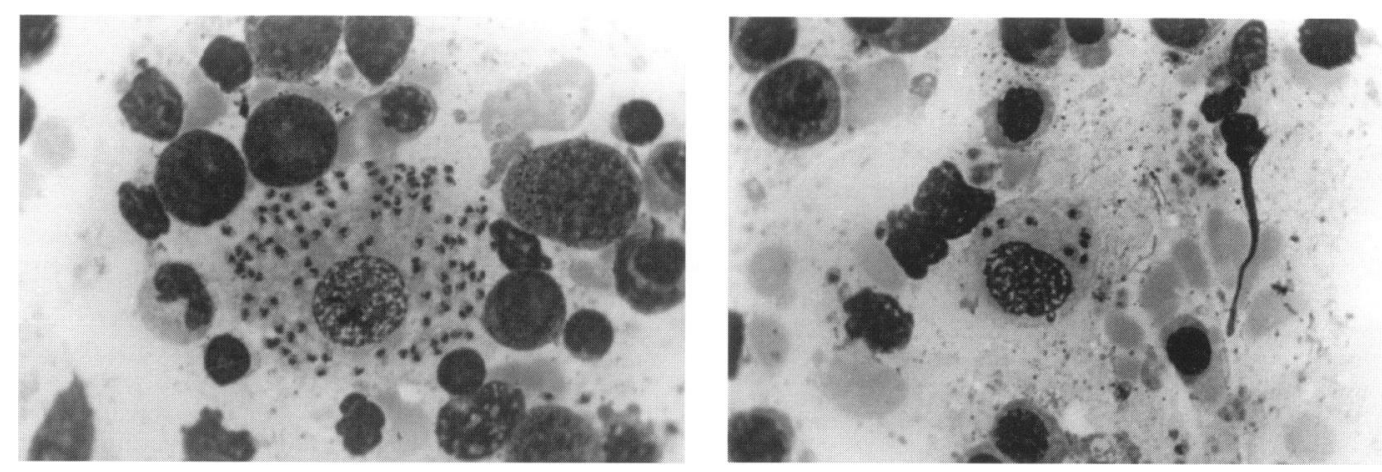

Figures 1 and 2 Macrophages containing intracellular micro-organisms in the bone marrow

Table Some laboratory tests

\section{Department of Adult Haematology, \\ Hacettepe University \\ Medical School, \\ Ankara, Turkey \\ Y Büyükaşık \\ IC Haznedaroğlu \\ H Demiroğlu, \\ $S$ Dündar}

\section{First Department of Internal Medicine, Numune Hospital, Ankara, Turkey N S İleri}

Correspondence to Yahya Büyükaşı, Sergenler Sok. Arzu Apt. 8/15 İç Cebeci 06590 Ankara, Turkey

Accepted 1 October 1997

\begin{tabular}{ll}
\hline Haemoglobin $(\mathrm{g} / \mathrm{l})$ & 72 \\
Red blood cell count $\left(\mathrm{x} 10^{12} / \mathrm{l}\right)$ & 3.0 \\
Leucocyte count $\left(\mathrm{x} 10^{9} / 1\right)$ & 2.4 \\
Platelet count $\left(\mathrm{x} 10^{\%} / 1\right)$ & 117 \\
Corrected reticulocyte ratio $(\%)$ & 3.5 (normal $0.5-2.5)$ \\
Erythrocyte sedimentation rate $(\mathrm{mm} / \mathrm{h})$ & 85 \\
Serum globulin concentration $(\mathrm{g} / \mathrm{dl})$ & 4.3 (normal $2.3-3.5)$ \\
Immunoglobulin G $(\mathrm{mg} / \mathrm{dl})$ & $4100($ normal $700-1500)$ \\
Serum immunoglobulin electrophoresis & Polyclonal hyperglobulinaemia \\
Antinuclear antibodies & Negative \\
Coombs tests, cold agglutinin & Negative \\
Thick and thin peripheral smears & Parasitaemia not identified; increase in the proportion of monocytes (12\%) \\
Bone marrow smear & Trilineage hyperplasia; many macrophages containing cytoplasmic microorganisms \\
Plain chest X-ray & Normal \\
Abdominal ultrasonography & Hepatosplenomegaly; normal liver paranchyma and portal venous system \\
Liver biopsy & Increased Kupfer cells containing cytoplasmic microorganisms \\
\hline
\end{tabular}

Blood and bone marrow cultures for non-specific microorganisms, brucellosis, salmonellosis and tuberculosis, and polymerase chain reaction analysis for mycobacterium tuberculosis in the bone marrow were all negative.

\section{Questions}

1 What is your diagnosis?

2 Summarise the geographical distribution of the disease.

3 What are the major complications of the disease?

4 What are the present drugs of choice in the treatment? 


\section{Answers}

QUESTION 1

Visceral leishmaniasis (kala azar)

QUESTION 2

Visceral leishmaniasis is endemic in many areas including the Mediterranean region, Southwest Asia, China, Latin America, East Africa, and India. ${ }^{1-3}$

QUESTION 3

Without treatment, visceral leishmaniasis is associated with a high rate of mortality (90-95\%), mostly due to secondary infections and haemorrhagic complications. Post kala azar dermal leishmaniasis, characterised by multiple hypopigmented (or erythematous) macules and massive lepromatous-like nodules on any part of the body, may also occur in some areas, generally one to several years after apparently successful treatment. ${ }^{13}$ Leishmaniae are present in these lesions, which are highly resistant to chemotherapy. The sufferers may act as a reservoir of Leishmania donovani.

\section{QUESTION 4}

Pentavalent antimony formulations are the drugs of choice: antimony $N$-methyl glucamine (meglumine antimoniate, Glucantime ${ }$, 20-60 $\mathrm{mg} / \mathrm{kg}$ per day intramuscularly) and sodium antimony gluconate (stibogluconate, Pentostam ${ }^{\circledR}, 10-20 \mathrm{mg} / \mathrm{kg}$ per day intramuscularly). ${ }^{12}$

\section{Discussion}

Visceral leishmaniasis results from multiplication of leishmaniae within the mononuclear phagocytes. The disease is primarily caused by Leishmania donovani in the Indian subcontinent and Africa, $L$ infantum in Mediterranean regions, and $L$ chagasi in Latin America. The pathogens exist in two forms: human disease is caused by the aflagellate form (amastigote) within the cytoplasm of the host macrophages (figures 1 and 2). The organism is transmitted to human by an insect vector, phlebotomine sandflies, in which it exists in the flagellate form (promastigote). Rodents, dogs, man, foxes and small carnivores are the principal reservoirs for different subspecies in various geographic areas. ${ }^{2}$ An irregular (anarchical) fever, hepatosplenomegaly, cytopenias, and emaciation, which occur after a variable incubation period from weeks to many years, are the principal signs of the infection. ${ }^{23}$ Confirmation of the diagnosis is based on three procedures: histological examination, serological studies, and in vitro cultivation of the parasites.

In addition to endemic areas, sporadic cases are also reported in many regions, including West European countries. These cases are mostly imported from the endemic areas, especially the Mediterranean countries due to popularity of Mediterranean holiday resorts. However, autochthonous cases have also been

\section{Infection-related haematological problems}

Destructive/consumptive cytopenias

- red blood cell invasion (malaria, bartonellosis, babesiosis)

- platelet invasion (malaria, trypanosomiasis)

- microangiopathic haemolytic anaemia with or without thrombocytopenia (disseminated intravascular coagulation, possible infectious aetiology in thrombotic thrombocytopenic purpura and haemolytic uraemic syndrome)

- cold agglutinin or Donath-Landsteiner antibody-induced immune haemolysis (mycoplasma, listeria, Epstein-Barr virus (EBV) syphilis, measles, kala azar, trypanosomiasis)

- infection-associated haemophagocytic syndrome (cytomegalovirus (CMV), EBV, Leishmania donovani, Candida albicans, tuberculosis, etc)

- release of haemolysins/direct erythrocyte toxicity (Clostridium perfringens sepsis, trypanosomiasis)

- oxidative damage in individuals with congenital erythrocyte enzyme deficiencies (glucose-6phosphate dehydrogenase, etc)

- immune thrombocytopenia (varicella, rubella, EBV, CMV, malaria, trypanosomiasis, etc, possible infectious trigger in idiopathic thrombocytopenic purpura)

- paradoxical leucopenia (toxic effect, increased utilisation, eg, sepsis)

Anaemia of chronic disease

Anaemia of acute infection (generally in children, uncertain pathogenesis)

Deficiency anaemias

- iron deficiency (ankylostomiasis, schistosomiasis)

- vitamin B12 deficiency (Diphyllobothrium latum)

Bone marrow suppression

- cytopenias due to infection of the progenitor cells (HIV, hepatitis $B$ virus)

- bone marrow infiltration/improper micro-environment (disseminated tuberculosis)

- aplastic anaemia (parvo virus B19 infection in patients with underlying haemolytic anaemia, hepatitis $\mathrm{C}$ virus, HIV, infectious mononucleosis)

Hypersplenism (malaria, visceral leishmaniasis, infective endocarditis, schistosomiasis, infectious mononucleosis, etc)

Bleeding and thrombosis (thrombocytopenia, disseminated intravascular coagulation, septic embolism in infective endocarditis)

Carcinogenesis (human T-cell leukaemia virus 1 causes adult T-cell leukaemia/lymphoma, EBV is implicated in Burkitt's lymphoma, increased risk of lymphoma in AIDS)

noted in Europe, mainly as an opportunistic infection during human immunodeficiency virus (HIV) infection. ${ }^{5}$

The response rate to antimony therapy in visceral leishmaniasis is between 87 and $98 \%$. Treatment failure is common in patients with HIV infection. ${ }^{5}$ In such cases, amphotericin B (preferably liposomal amphotericin B) can be used. ${ }^{1}$ Pentamidine, paromomycin and combinations of interferon- $\gamma$ and antimony preparations ${ }^{6}$ may also be considered as second choice agents. ${ }^{1}$ However, they are all likely to be less effective than amphotericin B. The present case dramatically improved with meglumine antimoniate $(60 \mathrm{mg} / \mathrm{kg}$ per day for 15 consecutive days). After seven days 
treatment, fever had disappeared and two months later, hepatosplenomegaly and pancytopenia had also resolved. The patient has been followed-up without any complication for two years.

1 Berman JD. Human leishmaniasis: clinical, diagnostic, and chemotherapeutic developments in the last 10 years. Clin Infect Dis 1997;24:684-703.

2 World Health Organization. The leishmaniases. Report of WHO expert committee. WHO Tech Rep Ser 1984; no 701

3 Bouree P, Belec L. Leishmaniasis: report of 33 cases and review of the literature. Comp Immun Microbiol Infect Dis $1993 ; 16: 251-65$.

\section{Final diagnosis}

Visceral leishmaniasis (kala azar).

Keywords: leishmaniasis; infection

4 Chance ML. The six diseases of WHO. Leishmaniasis. BMF 1981;283:1245-7.

5 Jeannel D, Tuppin P, Brucker G, Danis M, Gentilini M Imported and autochthonous kala-azar in France. $B M F$ 1991;303:336-8.

6 Badaro R, Falcoff E, Badaro FS, et al. Treatment of visceral leishmaniasis with pentavalent antimony and interferon gamma. N Engl f Med 1990;322:16-21.

\title{
An adolescent girl with abnormal liver profile
}

\author{
Satheesh Nair, C S Pitchumoni
}

A 17-year-old previously healthy high school student who lived in a dormitory was referred to our office by her private physician for evaluation of abnormal liver function tests. She was sexually active with one partner but denied any current or past substance abuse. The patient was not taking any medications or nutritional supplements. Family history was unremarkable. Physical examination revealed scleral icterus and minimal hepatomegaly. Spleen was not palpable. The liver function tests are shown in table 1 . Total leucocyte count was $6.3 \times 10^{9} / 1$ with $53 \%$ lymphocytes. The platelet count was normal. Anti-Hbc IgM antibody was negative, so were anti-HAV IgM and anti-HCV antibodies. HBsAg was negative and anti-HBs antibody was positive. Erythrocyte sedimentation rate was $16 \mathrm{~mm}$ in the first hour. An abdominal sonogram was done to evaluate a persistent elevation in alkaline phosphatase and it showed only hepatomegaly.

Table 1 Liver function tests

\section{Division of Medicine and Gastroenterology, Our Lady of Mercy Medical Center, New York Medical College, 600 East 233 Street, Bronx, NY 10466, USA S Nair C S Pitchumoni}

Correspondence to CS Pitchumoni

\begin{tabular}{|c|c|c|c|c|c|c|}
\hline \multirow[b]{2}{*}{ Duration of illness } & \multirow[b]{2}{*}{ Normal range } & \multirow[b]{2}{*}{ First visit } & \multicolumn{4}{|c|}{ Week } \\
\hline & & & 1 & 3 & 4 & 5 \\
\hline Total bilirubin (mg\%) & $0.2-1.2$ & 1.4 & 2.4 & 1.3 & 1.3 & 1.1 \\
\hline Alanine transaminase $(\mathrm{IU} / \mathrm{ml})$ & $7-49$ & 474 & 322 & 92 & 35 & 13 \\
\hline Aspartate transaminase (IU/ml) & $10-38$ & 470 & 179 & 59 & 31 & 19 \\
\hline Alkaline phosphatase (IU/ml) & $7-128$ & 253 & 494 & 310 & 179 & 117 \\
\hline
\end{tabular}

\section{Questions}

1 What other historical information is important in evaluating this patient?

2 What is the next step in establishing the diagnosis?

3 What is the management of this patient? 